Department of Animal Diseases,

Veterinary College, AL-Baath University, Syria.

\title{
ISOLATION OF CAMPYLOBACTER FETUS INFECTION IN SHEEP IN NORTH AREA OF SYRIA
}

(With One Table)

\author{
By
}

\section{ELEWE ; Y. AL-YASENO* and A. ARWANA**}

* Dept. of Infectious Diseases, Fac. Vet. Med. AL-Baath University. ** Dept. of Meat Hygiene, Fac. Vet. Med. AL-Baath University.

(Received at 20/11/2010)

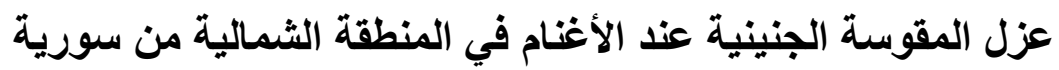

$$
\text { محد العليوي ، ياسبن الياسينو ، عبل العزيز عروانة }
$$

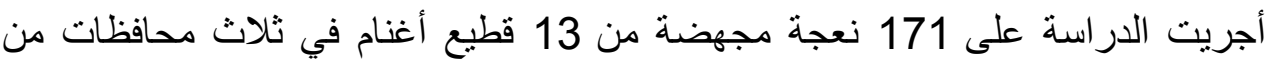

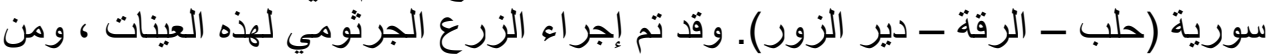

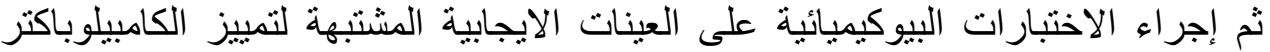

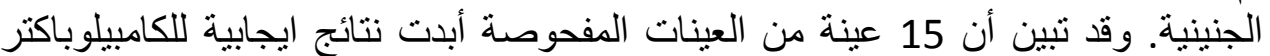

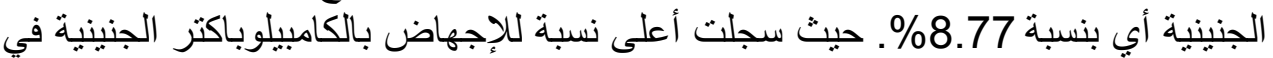
محافظة حلب 10.5\% ، بينما بلغت في محافظة الرقة نسبة الإجهاض بالكامبيلوباكتر الجنينية 6.9\%، أما في محافظة دير الزور فقد كانت النسبة 5.4\%. كما تضمن النها البحث المناقتشة العلمية لهذه النتائج ، وأهم الاستتناجات.

\section{SUMMARY}

This study has been carried out on 171 aborted ewes in 13 sheep folds in 3 Syrian provinces (Aleppo-Alraqa -Der alzor). The culture of these samples has been done, then the biochemical tests have been carried out on the positive samples to distinguish Campylobacter fetus. It has been proved that 15 samples have shown positive results for Campylobacter fetus which equals $8.77 \%$. The highest percentage of abortions caused by Campylobacter in Aleppo was $10.5 \%$, whereas it was $6.9 \%$ in Alraqa, the percentage was $5.4 \%$ in Der alzor. The research included the scientific discussion of this result, and the conclusions.

Key words: Campylobacter, sheep, abortion, fetus. 


\title{
INTRODUCTION
}

\author{
مقدم-ة
}

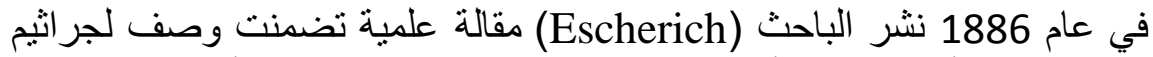

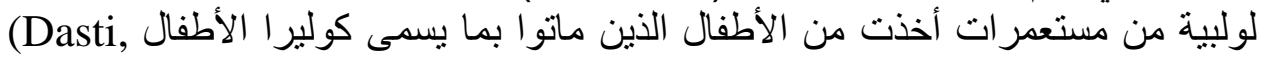

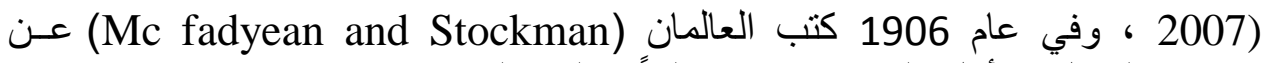

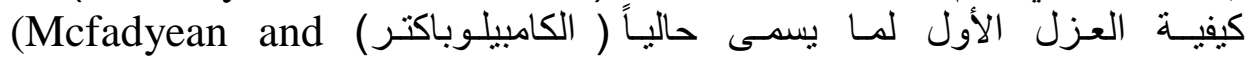
Stockman, 1913) إن الجراثيم المعروفة الآن بالكامبيلوباكتر عزلت بنجاح في بداية هذا القرن عام

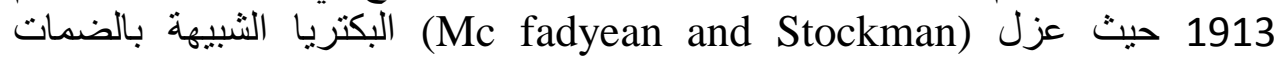
5 related Vibrio سنوات اكتثف (Smith) في عام 1918 بكتريـا لولبية في أجنة الأبقار المجهضة

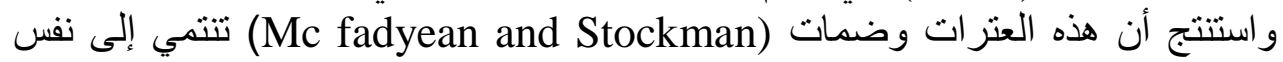

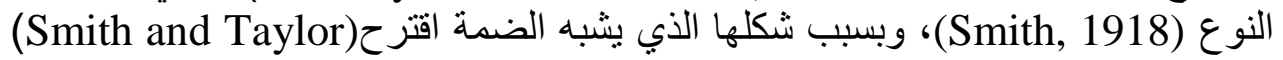

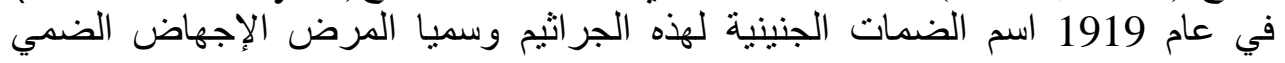
(Skirrow, 1977; Fox, 1982; Butzler,1984; Franco,1988) "vibrionic abortion"

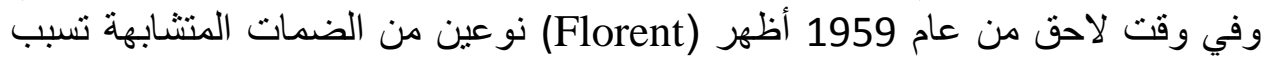

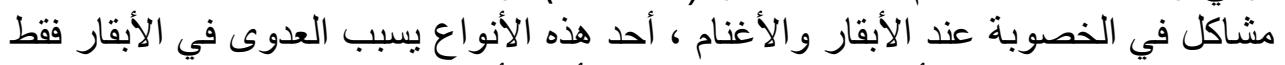

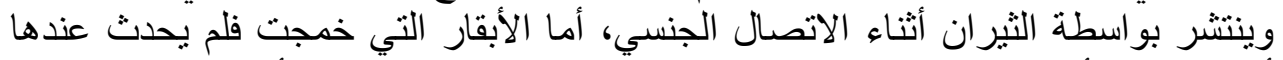

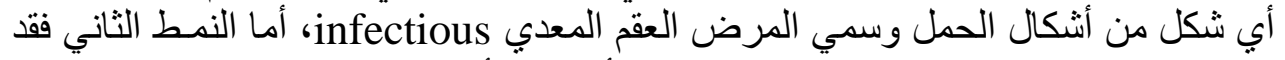

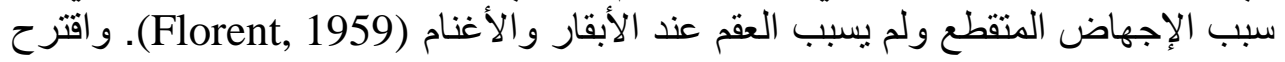
في عام 1973 م اسم الكامبيلوباكتر الجنينية تحت نوع (Veron and chatelaine)

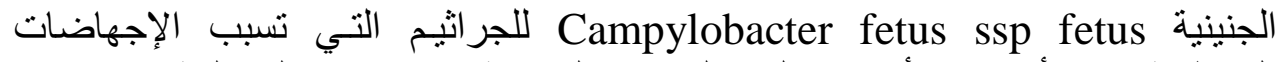

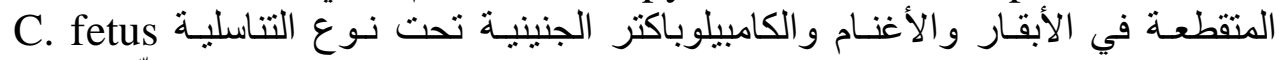
Ssp venerealis للجراثيم التي تسبب العقم المعدي (Prévot, 1940). شكّل جنس ونس Spirillum

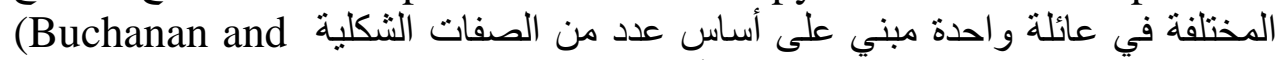

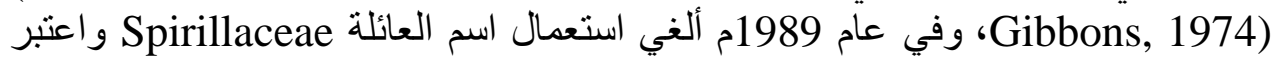

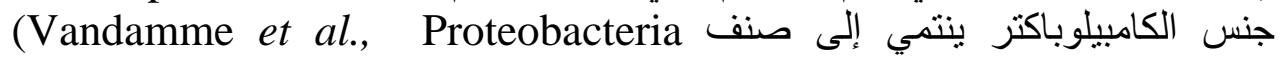

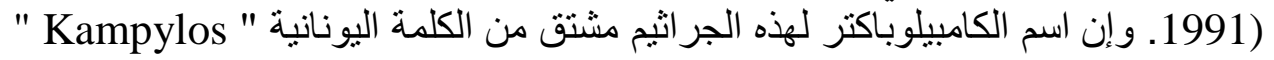

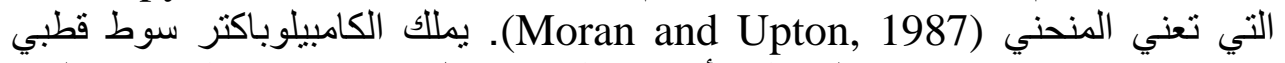
غير مغدد مفرد ( وحيد السوط) أو سوط في كل نهاية (سوطين منقابلين)

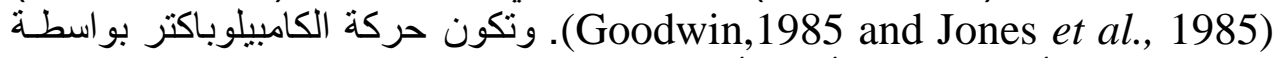

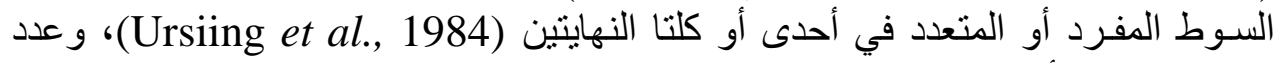

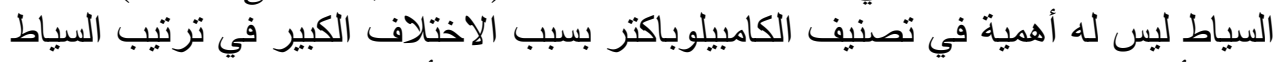

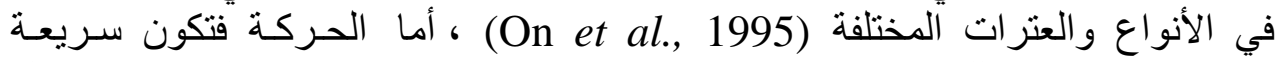
اندفاعية على هيئة اللولب (Karmali and Fleming, 1979). الكامبيلوباكتر الذي له له 


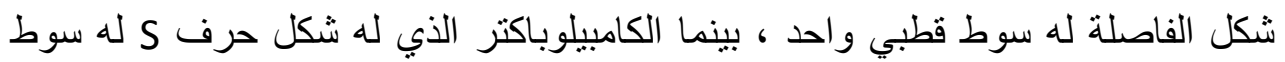

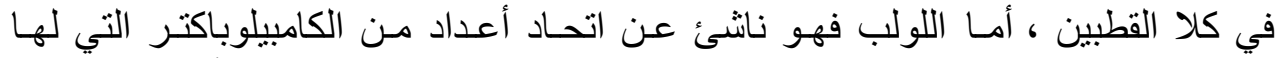
شكل S (McCoy et al., 1975). يكون النمو في ظروف لاهو النوائية أو قليلة الهواء وتتطلب هذه الجراثيم

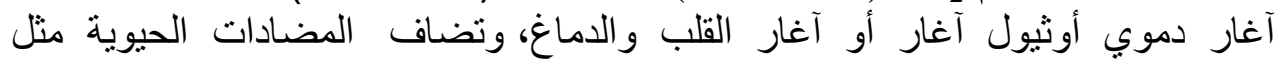

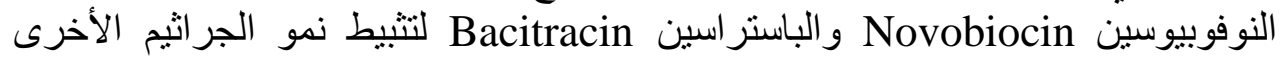

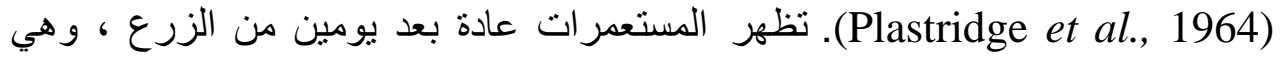

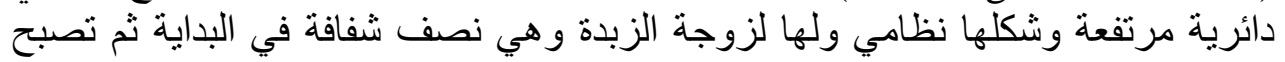

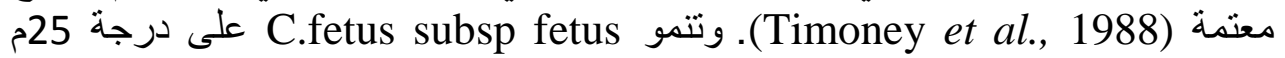
ويمكن أن تزرع على 37 م (Smibert and Von Graevenenitz, 1980). ويكون

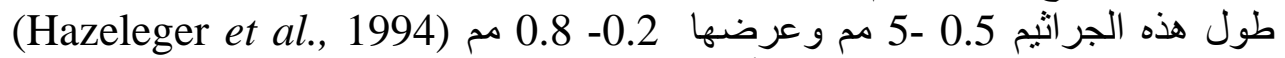
(Dyre, وتحصل العدوى عن طريق ابتلاع الأغنام للعلف والماء الملوثين بالعامل المسبب .2008)

ومن الممكن أن يكون طائر العقعق والغربان لهما دور في نقل العدوى وأيضاً

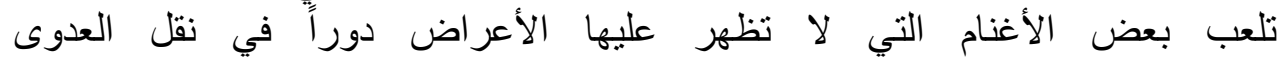
لهeinershagen et al., 1965; Smibert, 1965; Dennis, 1967) له أهية في تحديد مدى الإجهاض حيث أنه في العدوى التجريبية عند 105 يوم من الحمل

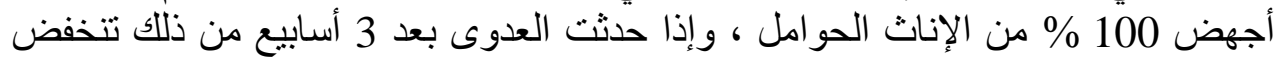

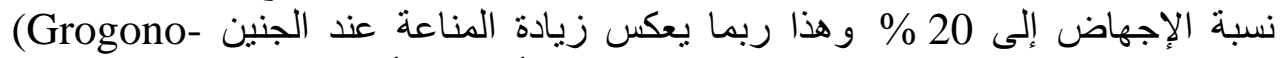
(Thomas et al., 2000)

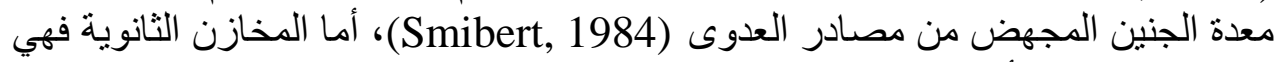

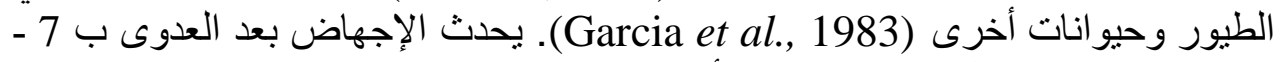

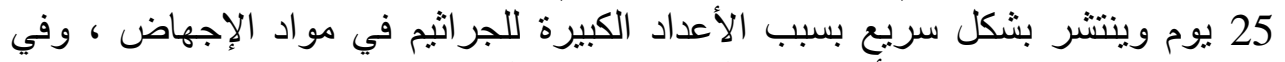

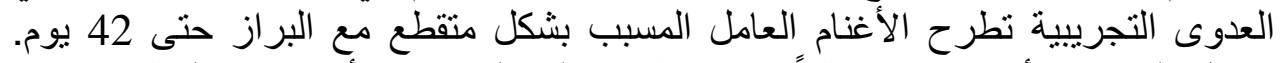

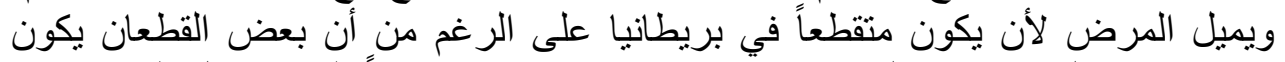

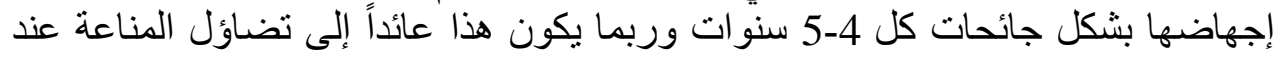

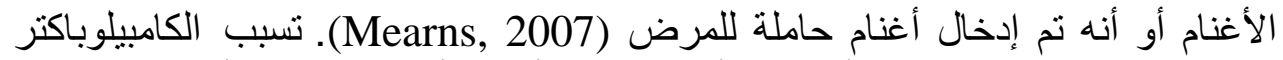

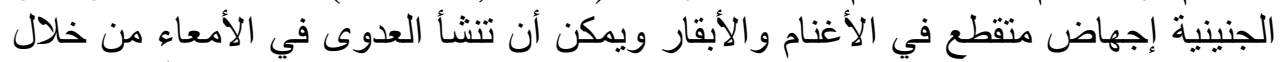

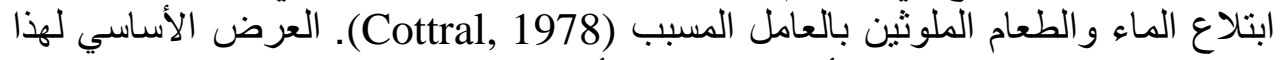

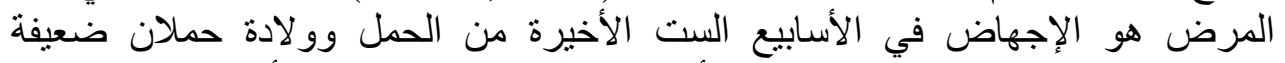

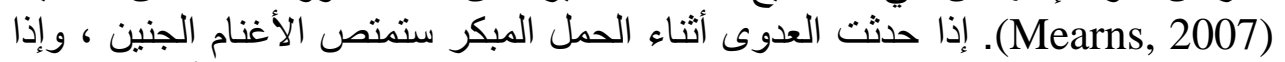

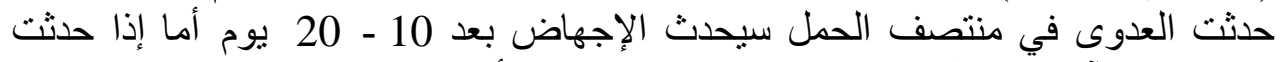

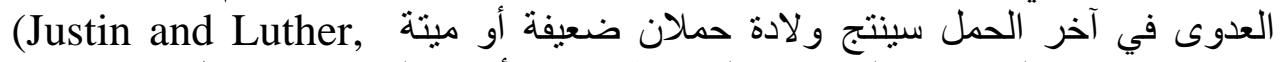

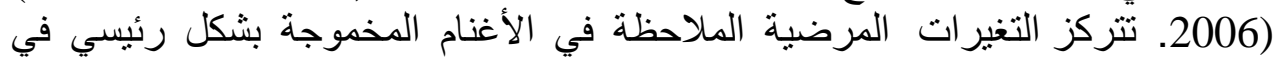

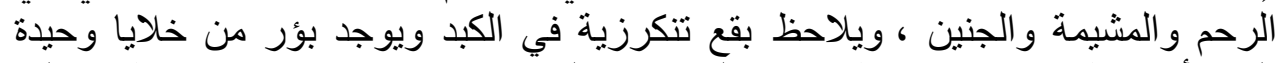

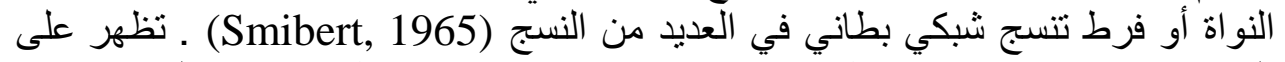
الكبد بؤر تنكرزية صفر اء قطر ها في ف-3 سم مع حواف في الفير منتظمة ويكون الكبد متضخم 
و هش و عليه بقع تنكرزية (Hum et al., 2009). تؤخذ العينات من المشيمة ومحتويات

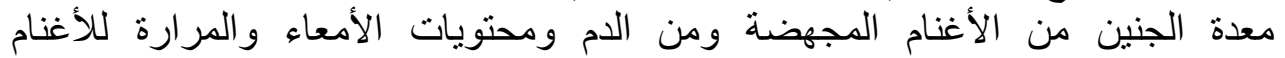

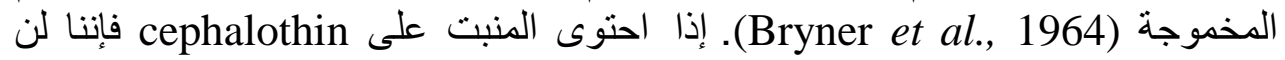

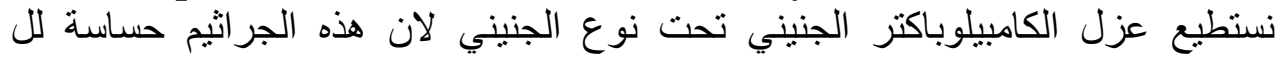
cephalothin

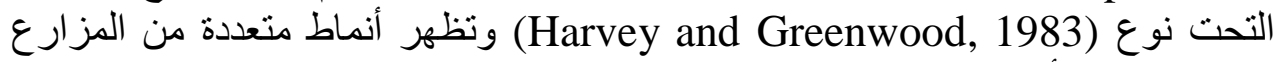

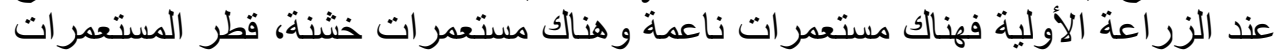

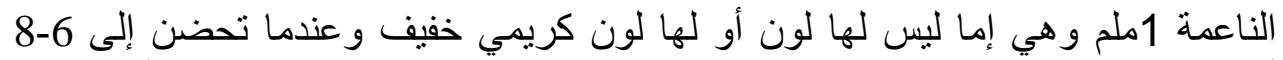

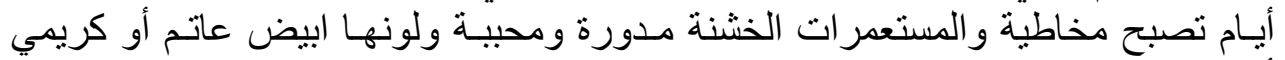

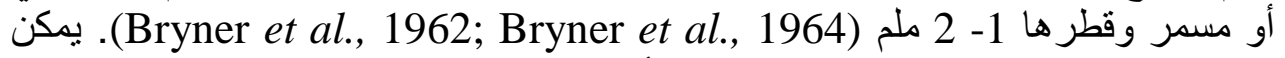

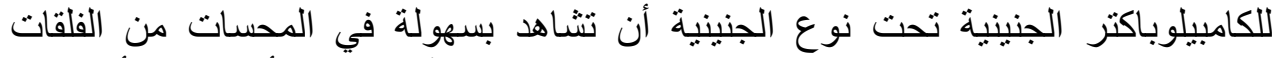

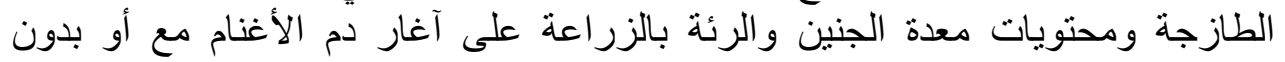

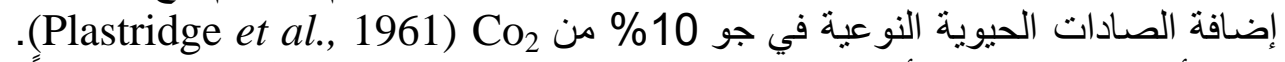
يمكن أن نستخدم تقنية الأجسام المضادة المشعة في التشخيص لكن ذلإن اللك يكون مستحيلاً

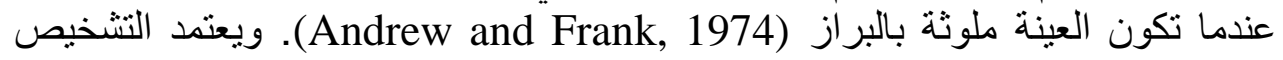

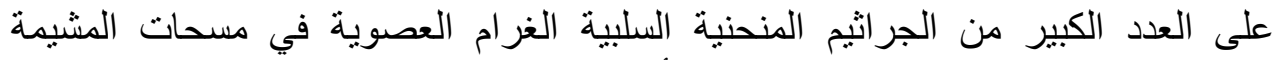

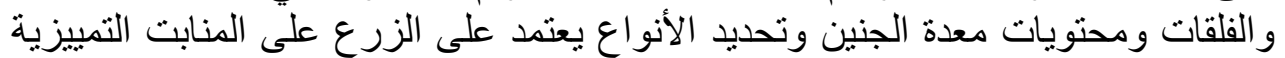

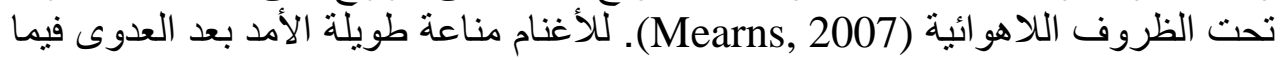

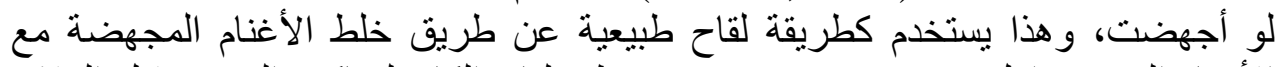

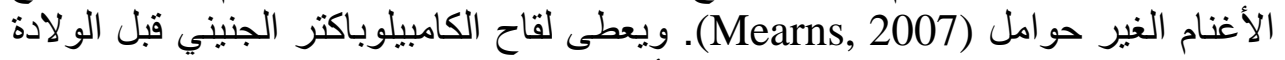

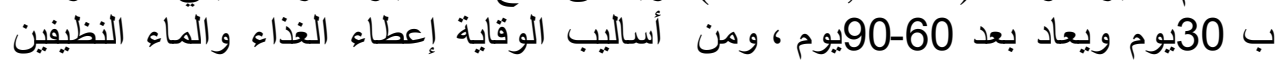
ووضع الأغنام التي ولات في مكان خاص (Justin and Luther, 2006). ويهدف البحث إلى عزل الكأمبيلوباكتر الجنينية عند الأغنام في محافظات المنطقة الثمالية

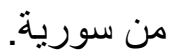

\section{MATERIALS and METHODS مواد وطر ائق البحث}

Samples collection : مئلاً

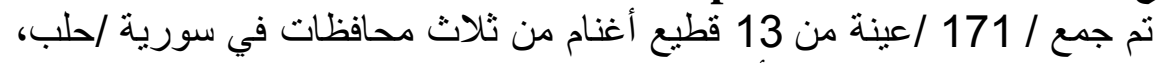

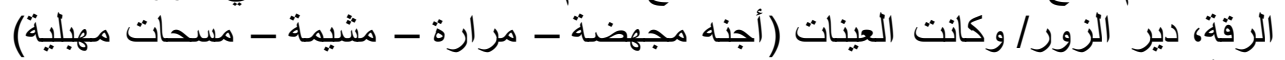

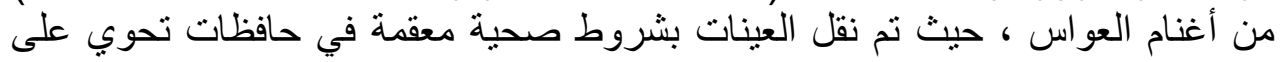
الجليد، ثم تم حفظها في درجة حرارة - 20م (Hum et al., 2009) حتى إجراء الاختبار ات اللازمة عليها. البيئات الزرعية الجرثومية: 1 - وسط سكيرو أغار الخاص بالكامبيلوباكتر : Skirrow's Campylobacter selective medium 
• Campylobacter Agar Base : وسط الكامبيلوباكتر اغار

Proteose Peptone

Liver digest

Yeast extract

Sodium chloride

Agar
$15 \mathrm{Gms} /$ litre

$2.5 \mathrm{~g} / 1$

$5 \mathrm{~g} / 1$

$5 \mathrm{~g} / 1$

$12 \mathrm{~g} / 1$

$\mathrm{PH}=7.4 \pm 0.2$

• المواد المضافة للمنبت التمبيزي للكامبيلوباكتر :

Campylobacter Supplement-III(Skirrow) كل أمبولة كافية ل500مل من الوسط

Polymyxin B $1.250 \mathrm{IU}$

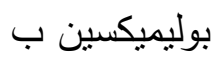

Vancomycin $\quad 5.00 \mathrm{Mg}$ فانكومايسين

Trimethoprim $2.500 \mathrm{Mg}$ تريمثوبريم

• دم أغنام غير مفبرن: Defibrinated sheep blood

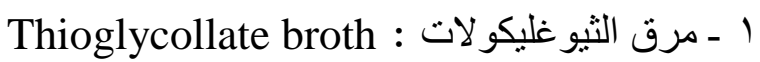

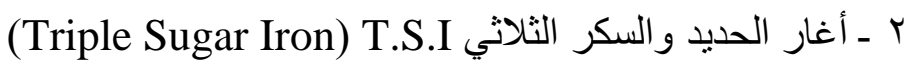

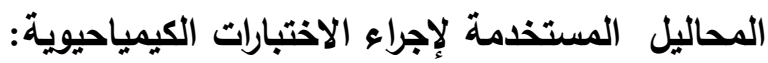

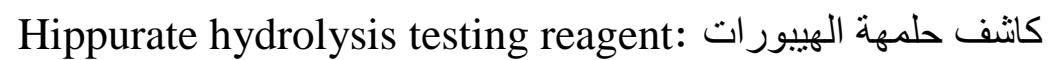

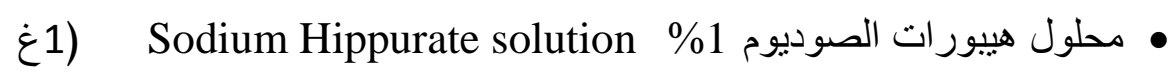

$$
\begin{aligned}
& \text { هيبورات الصوديوم و 100مل ماء مقطر ) } \\
& \text { يصب في كل أنبوب0.4 مل من المحلول } \\
& \text { ملمول الننهرين : Ninhydrine solution } \\
& \text { Ninhydrine } 3.5 \mathrm{~g} \\
& \text { Acetone } 50 \mathrm{ml} \\
& \text { • تنهرين } \\
& \text { Butanol } 50 \mathrm{ml} \\
& \text { - أسيتون }
\end{aligned}
$$

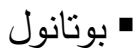

$$
\begin{aligned}
& \text { نمزج أولاً الأسيتون و البوتانول ثم نضيف لها الننهدرين }
\end{aligned}
$$

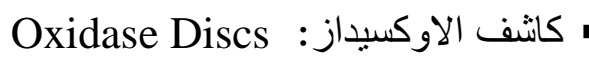

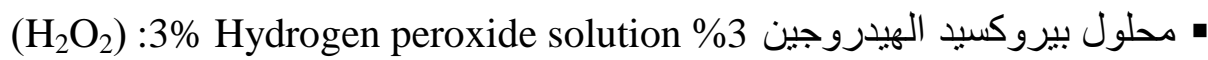




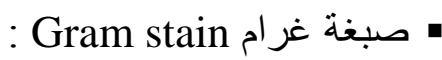

$$
\text { طرق العمل: }
$$

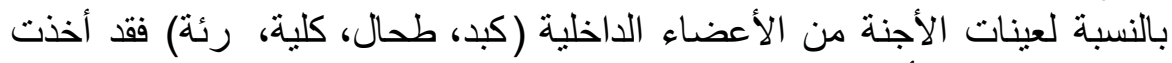

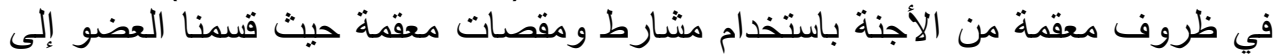

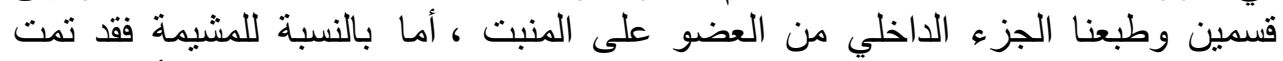
الزراعة من الفلقات التي تملك لون بني مصفر (Hum et al., 2009)، أما بالنسبة لمحتويات معدة الجنين والمسحات المهيلية وعينات المرارة فقد تم أخذ مسحات وزرعت

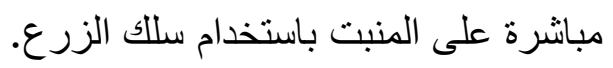

Plating on selective medium: الزرع على الوسط الانتقائي

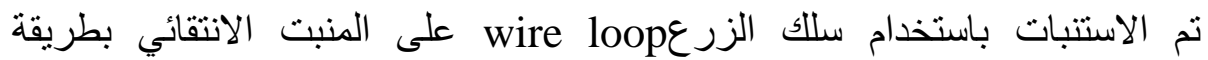

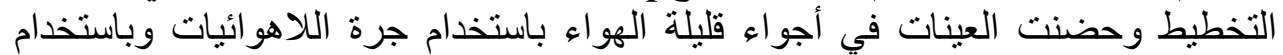

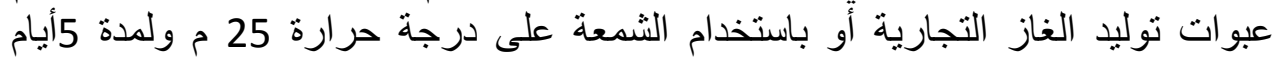

(OIE, 2008)

Identification of Campylobacter : التعرف على الكامبيلوباكتر

أولاً: الصفات المزرعية: تم فحص جميع المستعمرات النامية على أطباق بتري حيث

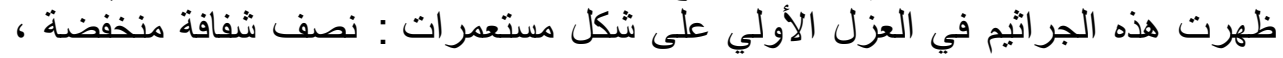

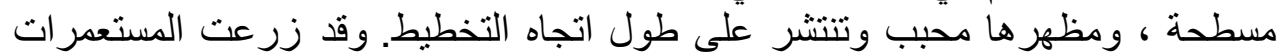

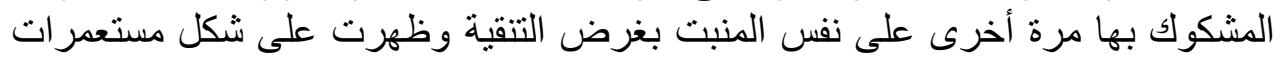
ناعمة ، محدبة ، بيضاء رمادية (أخرى (Bryner et al., 1962).

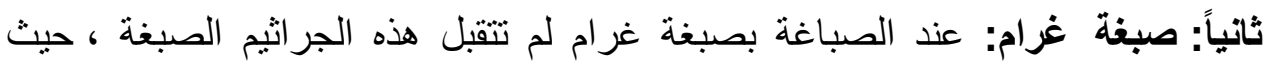

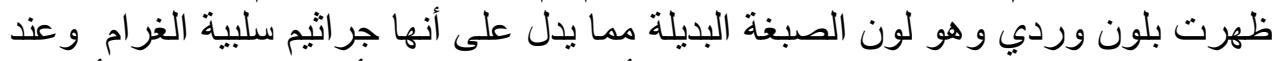

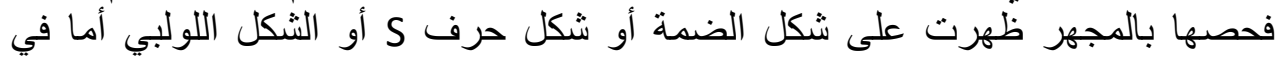
المزارع القديمة فإنها تميل للتكور (Smibert, 1974). ثالثاً: اختبار الحركة: أجري هذا الاختبار على المستعمر ات النّات النامية على المنابت الحديثة،

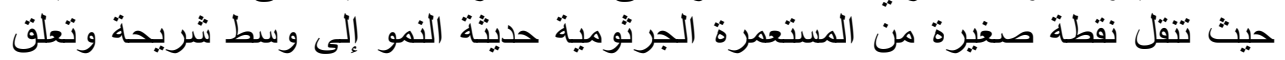

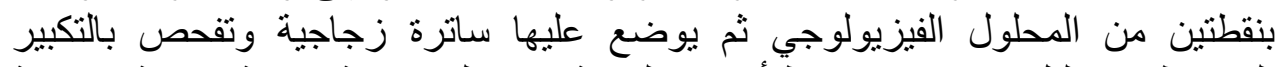

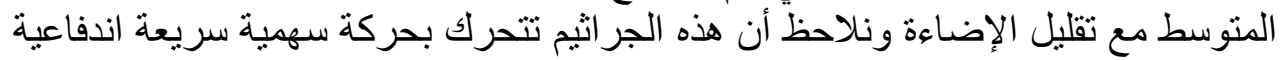

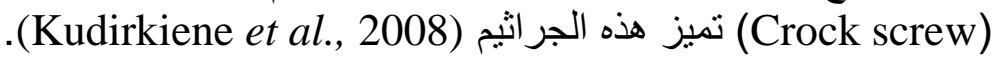
رابعاً: متطلبات الأوكسجين: هذه الجر اثيم قليلة الألفة للأوكسجين فهي تحتاج في نمو ها إلى الى .(Roshdy, 2000) (10\% $\mathrm{Co}_{2} 85 \% \mathrm{~N}_{2}$ ، $\left.5 \% \mathrm{O}_{2}\right)$

خامساً :الاختبارات الكيميا حيوية: Biochemical Tests 


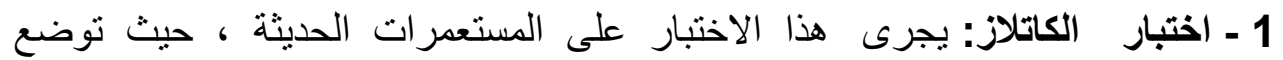

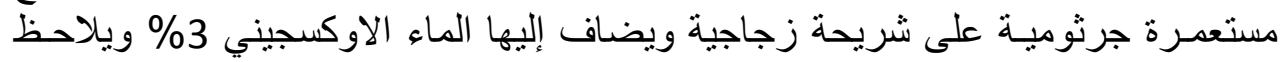

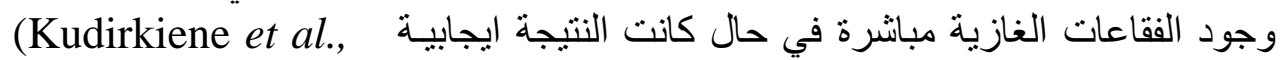

2 - اختبار الاوكسيداز: نأخذ قرص من أقراص الكاشف الجاهزة وتؤخذ المستعمرة

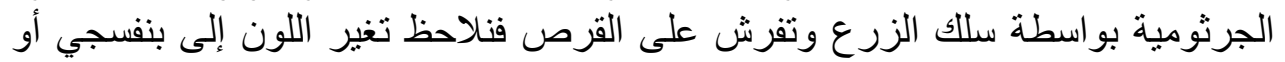
ازرق غامق خلال 10 دقائق (Kudirkiene et al., 2008).

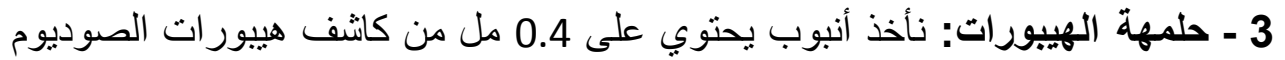

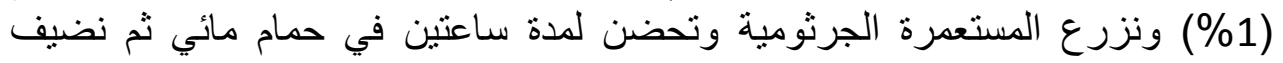

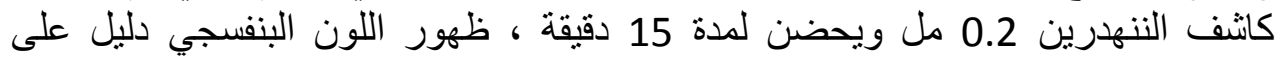
ايجابية الاختبار (Baron et al., 1994).

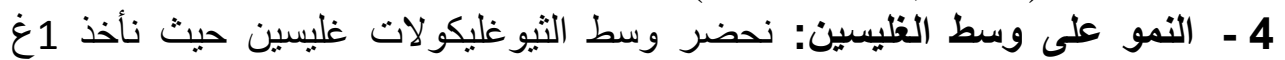

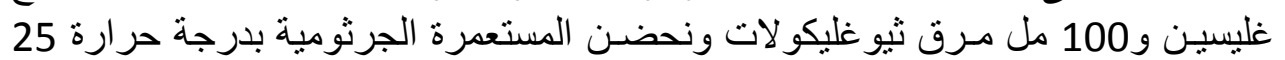

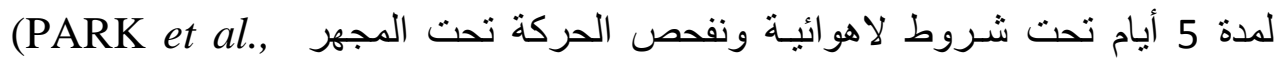
.1984)

سادساً :التحسس الجرثومي: Antibiotic sensitivity فرشت قطرة من معلق جرثومي على كامل سطح أطباق الكامبيلوباكتر اغار ووضع على سطح كل طبق أقراص مشبعة بالصادات الحيوية التالية: (10 IU) Penicillin

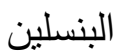
(10 mcg) Gentamycin

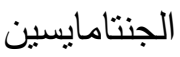
(30 $\mathrm{mcg})$ Neomycin النيومايسين

(15 mcg) Erythromycin

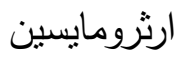
(30 mcg) Oxy Tetracycline اوكسي تتراسكلين (10 mcg) Streptomycin هتربتومايسين (5 mcg) Ciprofloxacin • سيبروفلوكساسين (30 $\mathrm{mcg})$

Nalidixic acid حض النالدكسك (30 mcg) Cephalothin السيفالوثين وبعد الحضن في درجة 25م لمدة 48 ساعة تم قياس قطر منطقة الصد حول

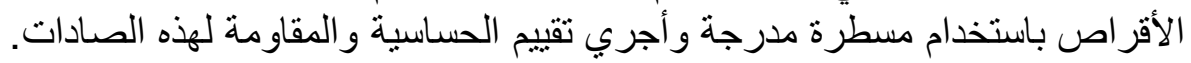

\section{RESULTS}




\section{النتائسج}

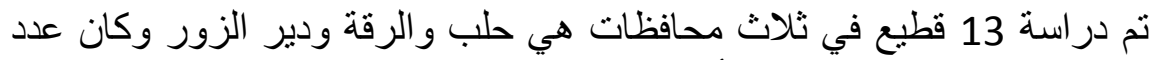

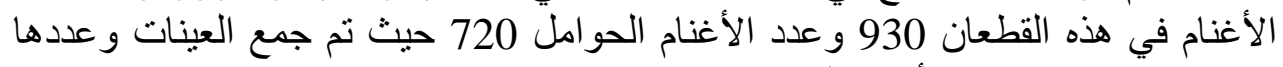
(171) عينة من جميع الأغنام المجهضة المعان. أما بالنسبة للأعراض الأنام المشاهدة فإن أهم عرض هو الإنة الإجهاض في الأسابيع

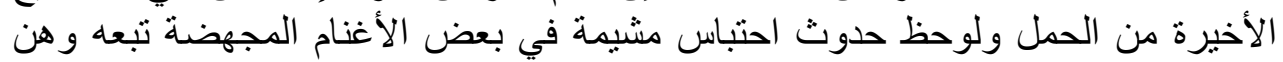
وضعف وذللك بسبب التهاب المجاري التناسلية الناتج عن العند العدوى الثان الثانوية.

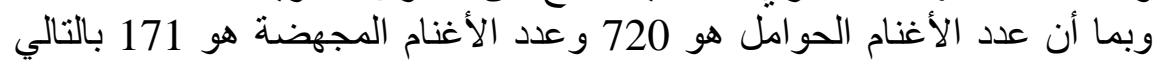

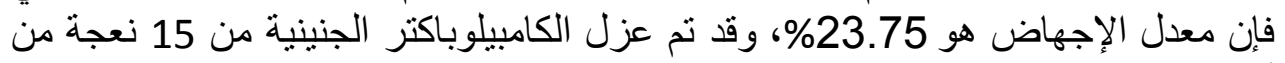

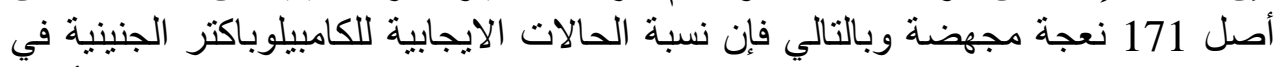

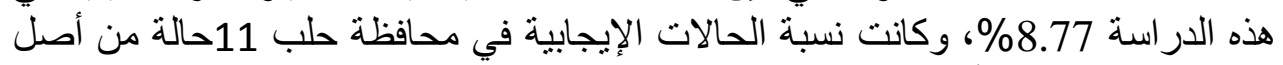

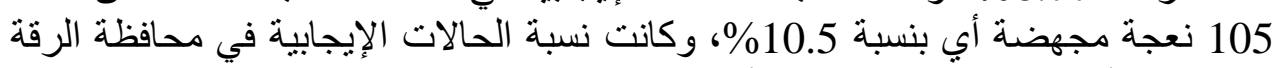
حالتين من أصل 29 نعجة مجهضة أي بنسبة 6.9\%ة وكاتب نهة وكانت نسبة الحالات الإيجابية في

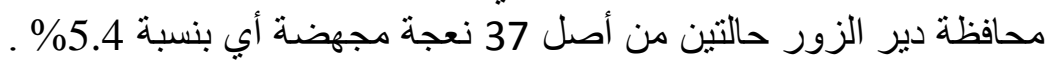

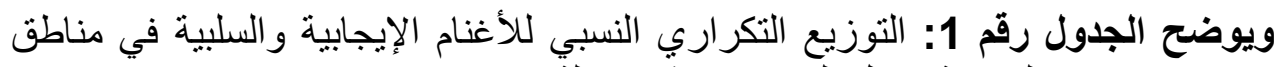

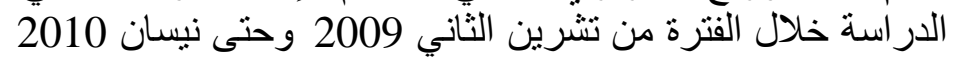

\begin{tabular}{|c|c|c|}
\hline التكرار التوزيعي المئوي للأغنام & معدل الإجهاض بالكامبيلوباكتر & المحافظة \\
\hline 89.5 & 10.5 & حلب \\
\hline 93.1 & 6.9 & الرقة \\
\hline 94.6 & 5.4 & دير الزور \\
\hline 91.23 & 8.77 & المجموع \\
\hline
\end{tabular}

تمت درأسـة الفروقات المعنوية بين معدلات الإجهاضـات وبين الحالات الإيجابيـة

التحليل الإحصائي

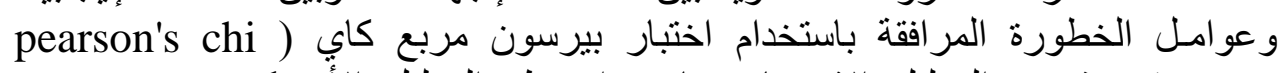

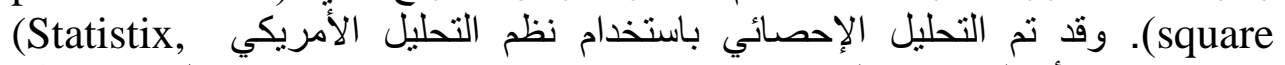

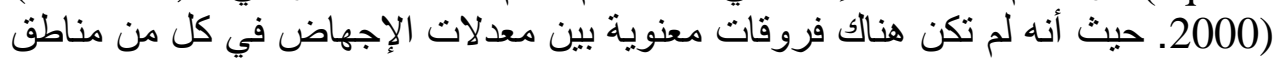

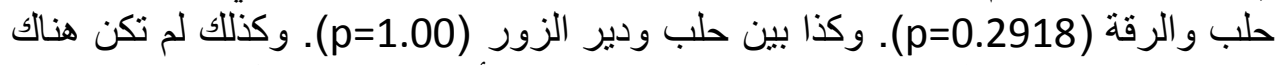

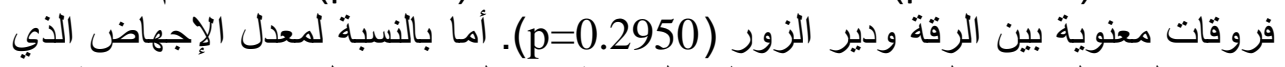

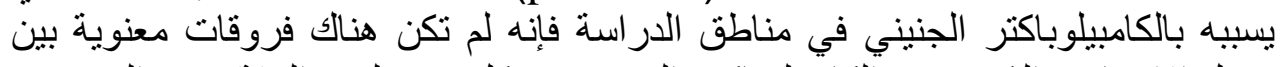

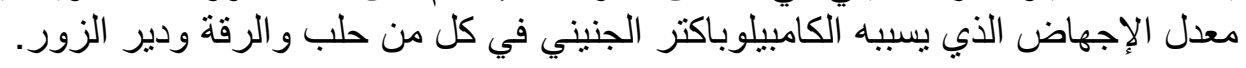

\section{DISCUSSION}

المناقثـة 


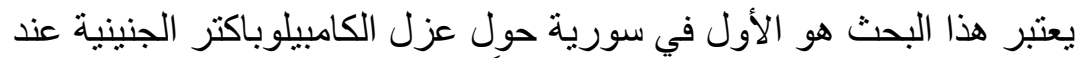

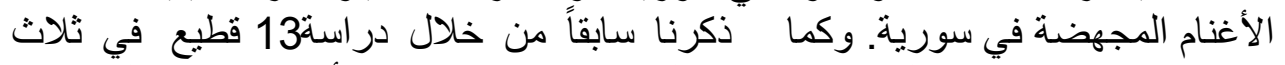

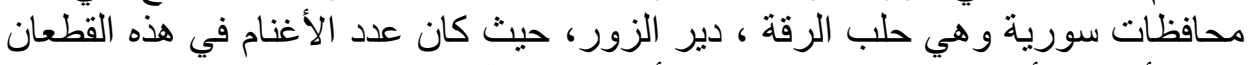

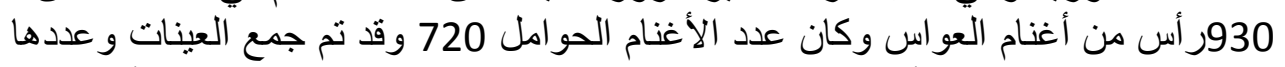

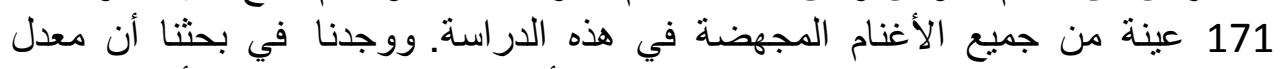
الإجهاض هو

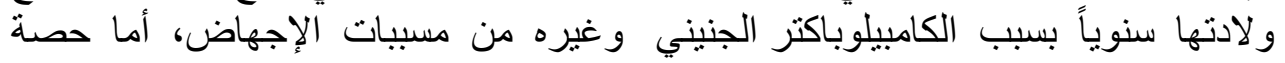

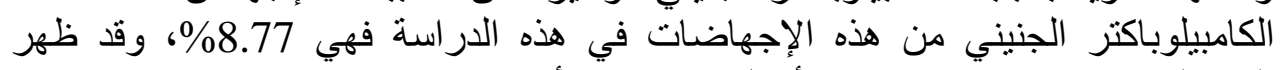

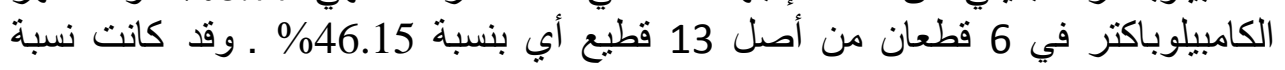

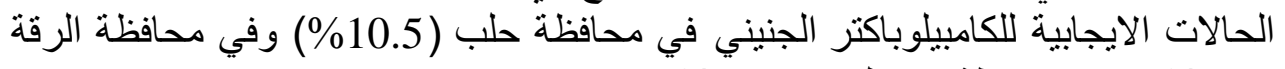

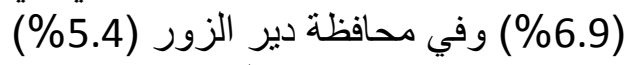

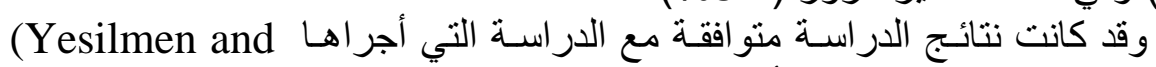

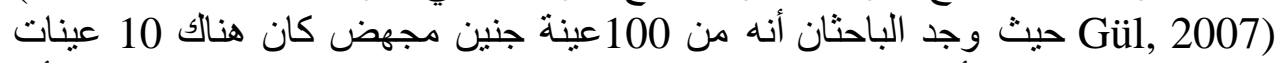

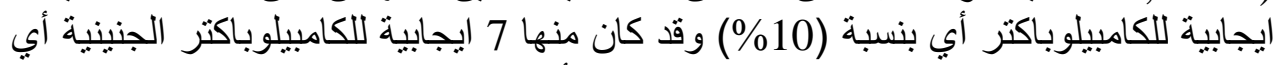

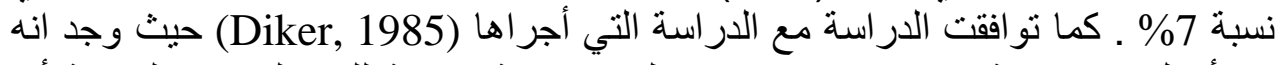

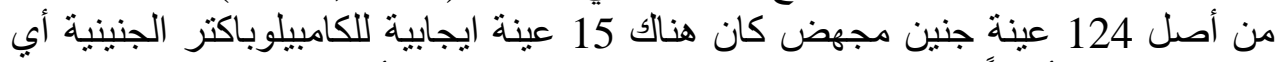

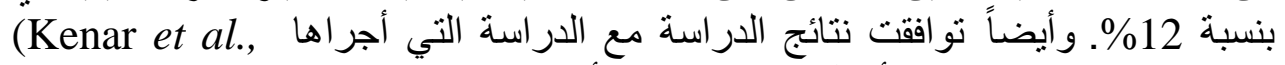

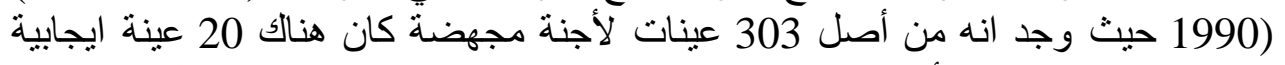

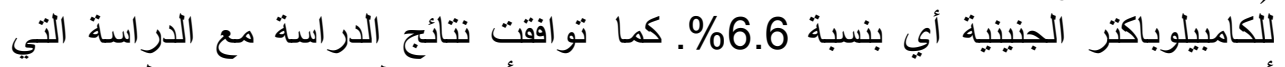

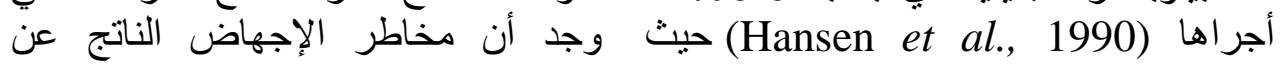

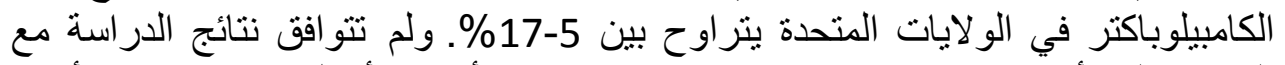

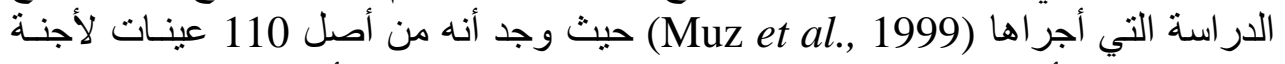

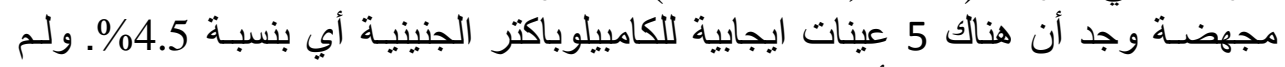

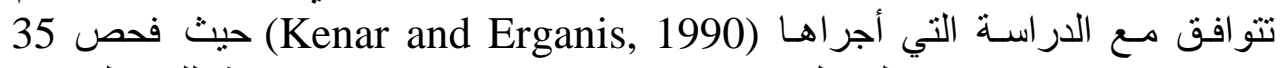
جنين مجهض في موسم التناسل الجرا 1991-1992 ووجد 5 عينات ايجابية للكامبيلوباكتر

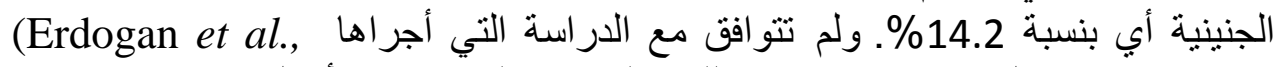

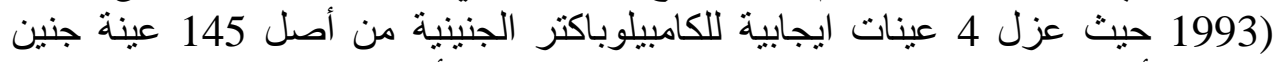

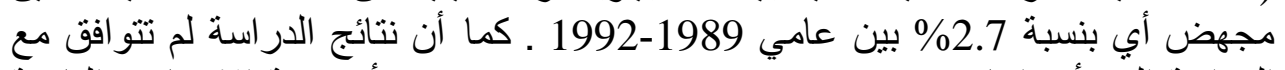

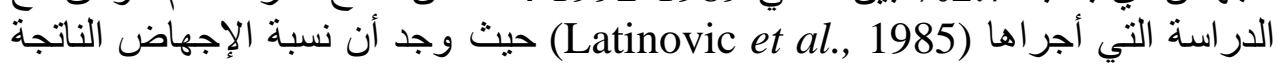

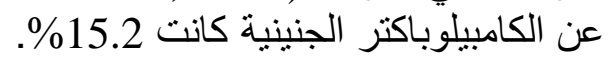

\section{CONCLUSIONS الاستتناجـات}

• تم عزل الكامبيلوباكتر الجنينية من الأغنام المجهضة في المنطقة الثمالية من سورية. • كانت نسبة الحالات الايجابية للكامبيلوباكتر الجنام الجنينية في الأغنام المجهضة الجنة في هذه

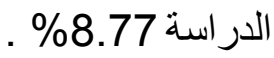


• بلغت نسبة الإجهاض في أغنام المنطقة الثمالية في هذه الدراسة 23.75 \%.

\section{REFERENCES}

Andrews, J. and Frank, F.W. (1974): Comparison of four diagnostic tests for detection of bovine genital vibriosis. J. Am. Vet. Med. Assoc 165: 695-697.

Baron, E.J.; Peterson, L.R. and Finegold, B.M. (1994): Baily and Scott's Diagnostic Microbiology. $9^{\text {th }}$ Ed. Mosby, St. Louis Baltimore.

Bryner, J.H.; O'Berry, P.A. and Frank, A.H. (1962): Dissociation studies of vibrios from the bovine genital tract. Am. J. Vet. Res. 23: 32-41.

Bryner, J.H.; O'Berry, P.A. and Frank, A.H. (1964): Vibrio infection of the digestive organs of cattle. Am. J. Vet. Res. 25: 1048-1050.

Buchanan, R.F. and Gibbons, N.F. (1974): Bergey's Manual of Determinative Bacteriology. 8th Edition. The Williams \& Wilkins Co., Baltimore.

Butzler, J. (1984): Campylobacter Infection in Man and Animals. CRC Press, Inc. Boca Raton, FL.

Cottral, G.E. (1978): Manual of standardized methods for veterinary microbiology, p. 461-471. Cornell University Press, Ithaca, N.Y.

Dasti, J.I. (2007): Identification and characterization of Campylobacter jejuni factors relevant for the infection process ph.D. Thesis, George August University, Germany.

Dennis, S.M. (1967): The possible role of the raven in the transmission of ovine vibriosis. Aust. Vet. J. 43: 45-48.

Diker, K.S. (1985): Studies on the identification of Campylobacter species isolated from sheep and cattle. DogaBilim. Derg. 9: 232-240.

Dyre, N.W. (2008): Diagnosis of Ovine Abortion - Getting the Most Out of Your Diagnostic Laboratory. Sheep Research Report: 13-14.

Erdogan, I.; Gurel, A.; Tekin, C.; Uyanýk, F. and Bitgel, A. (1993): The determination anddistribution of bacterial abortus in goats, cow and sheep in Thrace region. J. Pendik Vet. Microbiol., 24: 23-35.

Florent, A. (1959): Les deux vibriosis genitales: la vibriose duea V. fetus venerealis et la vibriose d'origine intestinaleduea V.fetus intestinalis. Meded. Veeartsenijsch. Rijksuniv. Gent 3: 1-60.

Fox, J.G. (1982): Campylobacteriosis: a "new" disease in laboratory animals. Lab. Anim. Sci, 32: 625-637.

Franco, D.A. (1988): Campylobacter species: considerations for controlling a foodborne pathogen. J. Food. Prot. 51: 145-153. 
Garcia, M.M.; Eaglesome, M.D. and Rigby, C. (1983): Campylobacters important in veterinary medicine. Vet. Bull. 53: 793-818.

Goodwin, C.S.; McCulloch, R.K.; Armstrong, J.A. and Wee, S.H. (1985): Unusual cellular fatty acids and distinctive ultrastructure in a new spiral bacterium (Campylobacter-pyloridis) from the human gastric mucosa. J. Med. Microbiol. 19: 257-267.

Grogono-Thomas, R.; Dworkin, J.; Blaser, M.J. and Newell, D.G. (2000): Roles of the surface layer proteins of Campylobacter fetus subsp. fetus in ovine abortion. Infect. Immun. 68: 1687-1691.

Hansen, D.E.; Hedstrom, O.R.; Sonn, R.J. and Synder, P.S. (1990): Efficacy of a vaccineto prevent Chlamydia or Campylobacter induced abortions in ewes. JAVMA, 196: 731-734.

Harvey, S.M. and Greenwood, J.R. (1983): Probable Campylobacterfetus subsp. fetus gastroenteritis. J. Clin. Microbiol. 18: 1278-1279.

Hazeleger, W.; Arkesteijn, Tooropbouma, A. and Beumer, R. (1994): Detection of the coccoid from or campylobacter jejuni in chicken products with the use of the polymerase chain reaction. Int. J. Syst. Bacteriol. 49: 1171-1175.

Hum, S.; Hornitzky, M. and Berg, T. (2009): Ovine campylobacteriosis. Elizabeth Macarthur Agricultural Institute. Australia:1-8.

Jones, D.M.; Curry, A. and Fox, A.J. (1985): An ultrastructural study of the gastric Campylobacter-like organism "Campylobacter pyloridis." J. Med. Microbiol. 131: 2235-2341.

Justin, S. and Luther, Ph.D. (2006): Abortions in Sheep Causes, Control and Prevention. NDSU. AS-1317.

Karmali, M.A. and Fleming, P.C. (1979): Campylobacter enteritis in children. J. Pediatr. 94: 527-533.

Kenar, B. and Erganis, O. (1990): Isolation and antibiotic susceptibility of Campylobacter spp. in aborted ovine fetuses in the central Black Sea. Veterinarium .5: 4-11.

Kenar, B.; Erganis, O.; Kaya, O. and Guler, L. (1990): Bacteriological and serological survey on brucella, campylobacter, salmonella and chlamydia infections caused to sheep abortion in Konya region (central Anatolia) in Turkey. Veterinarium, 1: 17-20 .

Kudirkiene, A.; Malakauskas, A.; Serniene, L. and Malakauskas, M. (2008): Isolation and identifaction of thermophilic Campylobacter ssp. by PCR-RFLP in broiler flocks. Veterinarija IR Zootechnik, A. 42(64): 44-46.

Latinoviç, V.; Popovic, M. and Nevjestic, A. (1985): Privi slucajevi izolacije bakterija roda Campylobacter kodgoveda i ovaca u Srbich. Veterinaria, Sarajevo, 34: 367-375. 
McCoy, E.C.; Doyle, D.; Wiltberger, H.; Burda, K. and Winter, A.J. (1975): Flagellar ultrastructure and flagella-associated antigens of campylobacter fetus. J. Bacteriol 122: 307-315.

Mcfadyean, J. and Stockman, S. (1913): Report of the Departmental committee appointed by the board of the Agriculture and Fisheries to inquire into Epizootic Abortion. part3. Abortion in sheep. London: HMSO.

Mearns, R. (2007): Campylobateriosis. pages131-132 In:Diseases of sheep ed, I.D. Aitken. Blackwell publishing, Oxford, U.K.

Meinershagen, W.A.; Waldhalm, D.G.; Frank, F.W. and Serivner, L.H. (1965): Magpies as a reservoir of infection for ovine vibriosis. J. Am. Vet. Med. Assoc.147: 843-845.

Moran, A.P. and Upton, M.E. (1987): Factors affecting production of coccoid forms by Campylobacter jejuni on solid media during incubation. J. App. Bacteriol 62: 527-537.

Muz, A.; Ertas, H.B.; Ongor, H.; Gulcu, H.B.; Ozer, H.; Eroksuz, H.; Dabak, M.; Basbug, O. and Kalender, H. (1999): Bacteriologic, serologic and pathologic studies onaborted cases of goats and sheep in Elazýð and it.s vicinity. Tr. J. Vet. Anim. Sci., 23: 177-188.

OIE "Organismo Internacional de Epizootias" (2008): Bovine genital campylobacteriosis chapter 2. 4. 5.

On, S.L.W.; Bloch, B.; Holmes, B.; Hoste, B. and Vandamme, P. (1995): Campylobacter hyointestinalis subsp. lawsonii subsp. nov., isolated from the porcine stomach, and an emended description of Campylobacter hyointestinalis. International J. Systemat. Bacteriol 45: 767-774.

PARK, C.E.; Smibert, R.M.; Blaser, M.J.; Vanderzant, C. and Stern, N. (1984): Campylobacter In: "Compendium of Methods for the Microbiological Examination of Foods "2nd Ed. Speck. M(ed) American public Health Association, Washington, D.C.

Plastridge, W.N.; Koths, M.E. and Williams, L.F. (1961): Antibiotic mediums for the isolation of vibrios from bull semen. Amer. J. Vet. Res. 22: 867-869.

Plastridge, W.N.; Stula, E.F. and Williams, L.F. (1964): Vibrio fetus infection and reinfection in heifers.as determined by cultural tests using blood agar plus antibiotics. Am. J. Vet. Res. 25: 710-713.

Prévot, A.R. (1940): Etudes de systématiquebactérienne. V. Essai de classification des vibrions anaérobies. Ann. Inst. Pasteur (Paris) 64: 117-125. 
Roshdy, M.S.A. (2000): Prevalence of Campylobacter jejuni in raw milk and infantile diarrhoea in Assiut. Master Degree Thesisi, Fac. of Med. Assiut University.

Senok, A.C. and Boota, G.A. (2009): CAMPYLOBACTER ENTERITIS IN THE Arabian Gulf. J. infect Developing Countries. 3(2):74-82.

Skirrow, M.B. (1977): Campylobacter enteritis: a "new" disease. Br. Med. J. 2: 9-11. Smith, T.; Orcutt, M.L. (1927). Vibrios from calves and their serological relation to Vibirio fetus. J. Exp. Med. 45: 391-397.

Smibert, R.M. (1984): Genus Campylobacter, p. 111-118. In N.R. Krieg, and H.G. Holt (ed.), Bergey's manual of systematic bacteriology, vol. 1. The Williams \& Wilkins Co., Baltimore.

Smibert, R.M. (1974): Genus II. Campylobacter Sebald and Veron 1963, 907, p. 207-212. In R.E. Buchanan and N. E.Gibbons (ed.), Bergey's manual of determinative bacteriology, $8^{\text {th }}$ ed. The Williams \& Wilkins Co., Baltimore.

Smibert, R.M. (1965): Vibrio fetus var. intestinalis isolated from the faecal and intestinal contents of clinically normal sheep: Biochemical and cultural characteristics of micro aerophilic vibrios isolated from the intestinal contents of sheep. Am. J. Vet. Res., 26: 320-327.

Smibert, R.M.; Von Graevenenitz, A. (1980): A human strain of C. fetus spp. intestinalis grown at $42^{\circ} \mathrm{C}$. J. Clin. Pathol. 33: 603-64.

Smith, T. (1918): Spirilla associated with disease of the fetal membranes in cattle (infectious abortion). J. Exp. Med. 28: 701-719.

Statistix (2000): Manual Guide. Version 4.0, Microsoft Co. Ltd. USA.

Timoney, J.F.; Gillespie, J.H.; Scott, F.W. and Barlough, J.E. (1988): Hagan and Bruner's microbiology and infectious diseases of domestic animals, $8^{\text {th }}$ ed., pp. 153-160. Cornell University Press, Ithaca, NY.

Ursing, J.B.; Lior, H. and Owen, R.J. (1994): Proposal of minimal standards for describing new species of the family Campylobacteraceae. Int J. Syst Bacteriol 44: 842-845.

Vandamme, P.; Falsen, E.; Rossau, R.; Hoste, B.; Segers, P.; Tytgat, R. and De Ley, J. (1991): Revision of Campylobacter, Helicobacter, and Wolinella taxonomy: emendation of generic descriptions and proposal of Arcobacter gen. nov. Int. J. Syst. Bacteriol. 41: 88-103.

Yesilmen, S. and Gül, K. (2007): Isolation, identification and antibiotic susceptibility of Campylobacter spp. in aborted sheep fetuses Medycyna Wet, 63: (10). 
Assiut Vet. Med. J. Vol. 57 No. 128 January 2011 